\title{
SOFT X-RAY LASER MICROSCOPE
}
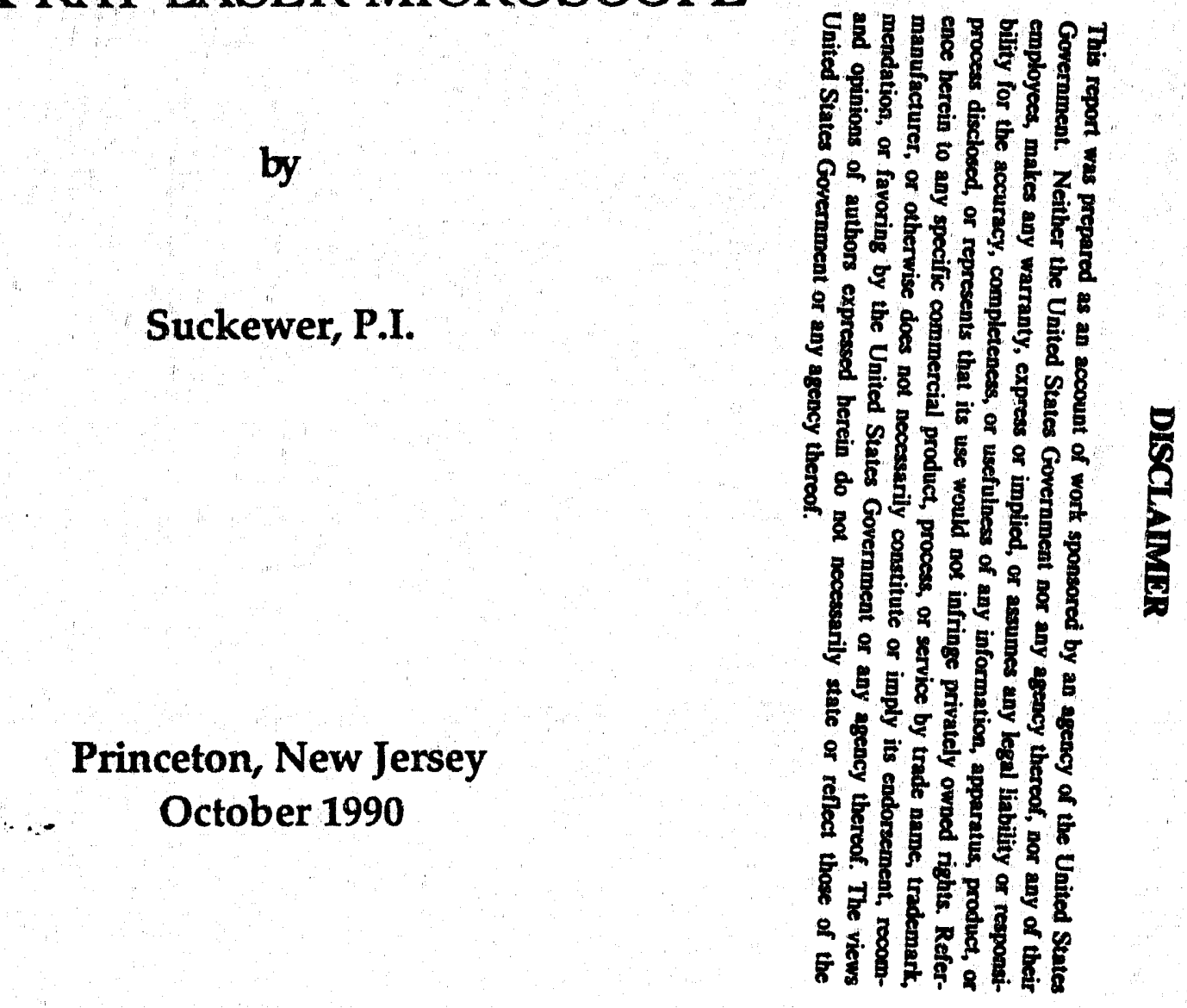

\section{MASTER}


Table of Contents

Page

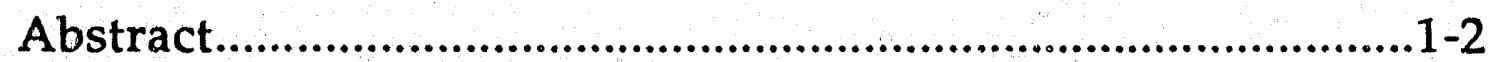

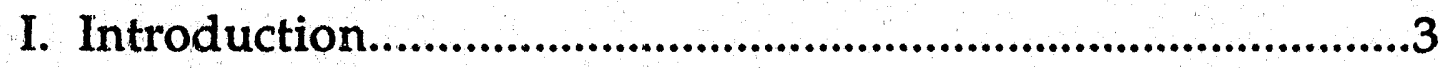

II. Simplified X-Ray Contact Microscope (Phase II)....................4-6

III. Composite Optical/X-Ray Laser Microscope (COXRALM) and Images of Biological Cells............................................... 7-9

IV. Imaging X-Ray Laser Microscope(IXRALM)....................10-11

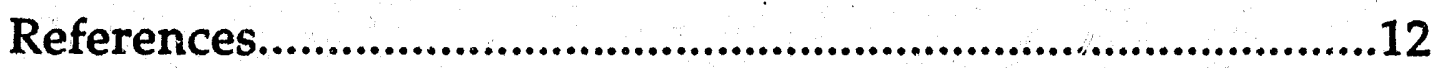

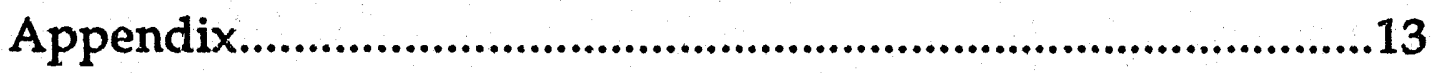


Final Report on

Soft X-Ray Laser Microscope

\begin{abstract}
The program consisted of two phases (Phase I and Phase II). The goal of the Phase I (first year program) was to design and construct the Soft X-ray Laser Contact Microscope. Such microscope was constructed and adapted to PPPL's $18.2 \mathrm{~nm}$ soft $X$ ray Laser (SXL), which in turn was modified and prepared for microscopy experiments. Investigation of the photoresist response to $18.2 \mathrm{~nm}$ laser radiation and transmissivity of $0.1 \mathrm{~m}$ thick silicionNitride (Si3 N4) windows were important initial works.
\end{abstract}

The goal of the first year of Phase II was to construct X-ray contact microscope in combination with existing optical phase microscope, already used by biologists. This Composite Optical XRay Laser Microscope (COXRALM; Award RD-100 for 1989) allows biologists to choose appropriate biological cells and make initial observations with optical microscope, followed by the $X$-ray image of chosen cells on photoresist using $X$-ray laser beam. This way approximately 5-10 times higher resolution than with optical microscope was obtained when "foot prints" on photoresist were analyzed with Scanning Electron Microscope (SEM).

In the second year of Phase II study of dehydrated Horseshoe Crab and Hela cancer cells were performed with COXRALM. Also during Phase II, the Imaging X-Ray Laser Microscope (IXRALM) was designed and constructed. This first design and construction employed microzone-plate and multilayer-coated Schwarzschild objective. Schwarzschild objective played a role of the condensor and zoneplate was used as an objective. System was designed for magnification $\approx 100$, which allows to use film or CCD array as a detector. IXRALM also incorporated additional Schwarzschild objective, identical to condensor ones, which creates interferometric 
system for alignment laser beam and condensor. However, we findout it is a very difficult task to properly align all optical system of the microscope, particularly microzone-plate. Therefore in the last part of Phase II, we re-designed the microscope in order to replace zoneplate by a second multilayer coated Schwarzschild objective, identical to the first one (condensor). Both Schwarzschild objectives were constructed, covered with multilayer coating and microscope was assembled. This microscope will be aligned and tested on the $X$ ray laser in Phase III, which will be done in cooperation between PXL and Princeton University (PU). 


\section{Introduction}

Princeton X-Ray Laser (PXL) in cooperation with Princeton University's Plasma Physics Laboratory conducted two phase programs (Phase I and Phase II) on the development of the X-ray laser microscopy. This cooperation was very fruitful and resulted in the designing and construction of three types of $X$-ray laser microscopes.

(a) Simplified X-Ray Contact Microscope (SIXCOM)

(b) Composed Optical/X-Ray Laser Microscope (COXRALM)

(c) Imaging X-Ray Laser Microscope (IXRALM)

Until now, the most important results from the point-of-view of the demonstration of capability of the X-ray laser microscopy, were obtained with the contact microscope COXRALM. COXRALM which was already build by PXL as a semi-commerical X-ray contact microscope. was used in the experiments related to the study of the immune system of the Horseshoe Crab cells (one of the most simple immune systems, which has a number of features similar to the human immune system) and cancer HeLa cells. These experiments were conducted in close cooperation with biologists. PXL and PPL (PU) were awarded RD-100 in 1989 for the development of COXRALM.

The most promising potential for biologists application of the $X$-ray laser microscopy is with IXRALM, which should be able to provide instantaneous, after the $X$-ray beam exposure, image of the biological cells on monitor screen, when CDD array is used as a twodimensional detector.

The next three sections describe the development of each microscopes and their application for research. 


\section{Simplified X-Ray Contact Microscope (Phase II)}

The development of the Simplified X-Ray Contact Microscope (SIXCOM) was conducted in Phase I of the program from September 15, 1986 until November 14, 1987. During this period, the main goal was to construct and use the SIXCOM on PPPL's Soft XRay Laser for initial studies of windows, resists, intensity requirements, achievable contrasts of biological material at $18.2 \mathrm{~nm}$, and attenuation losses of $\mathrm{Si}_{3} \mathrm{~N}_{4}$ window material. Princeton X-Ray Laser (PXL) was also used the contact $X$-ray microscope to gain operational experience on the $X$-ray laser-contact microscope system and, as well to develop cooperation with the biologists and physicists in order to broaden the applications of the X-ray laser microscopy. As a step in this direction, PXL has established a close collaborative relationship with the optical physicist (Prof. J Hirschberg) and biologist (Prof. E. Kohen) both working in the field of cancer research at the University of Miami. In collaboration with these researchers, PXL developed a Composite Optical/X-Ray Laser Microscope (COXRALM) for the study of cancer cells and immune system which will be described below.

PXL have also established collaborative relations with several leading researchers in $X$-ray microscopy: Drs. Chen, Feder and Sayre from IBM, and Prof. J. Kirz from the University of Stony Brook. Due to the advice and help from these researchers with the design of the contact microscope, we were not only able to omit a number of mistakes and make good progress with the construction of the microscope but also provide the necessary modifications to the soft X-ray laser beam focussing system for the contact microscope.

In Fig. I i shown schematically the set up of the soft X-ray laser with the contact microscope attached (see also Photo in Fig. 2). The laser beam is directed to the microscope by a remote controlled grazing incidence toroidal mirror (Photo in Fig. 3). In the first experiments a \#100 wire mesh as a test object was used and images were recorded in vacuum on Kodak 101 film and copolymer resist. 
co

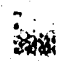

옹

马्ञ

है

恣

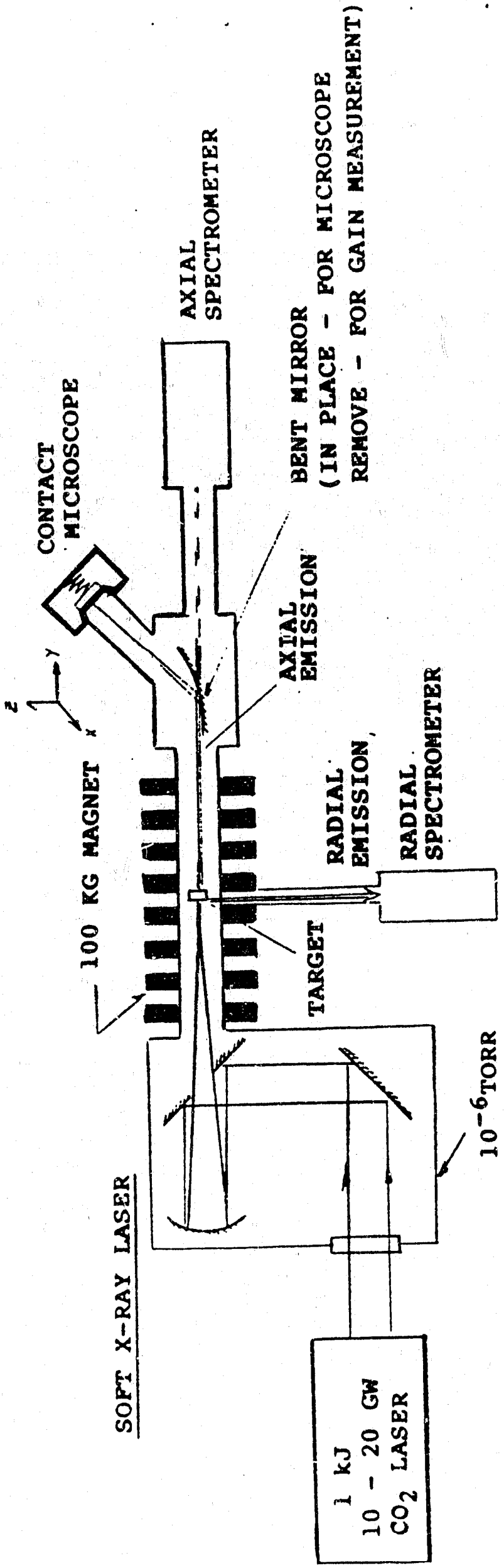




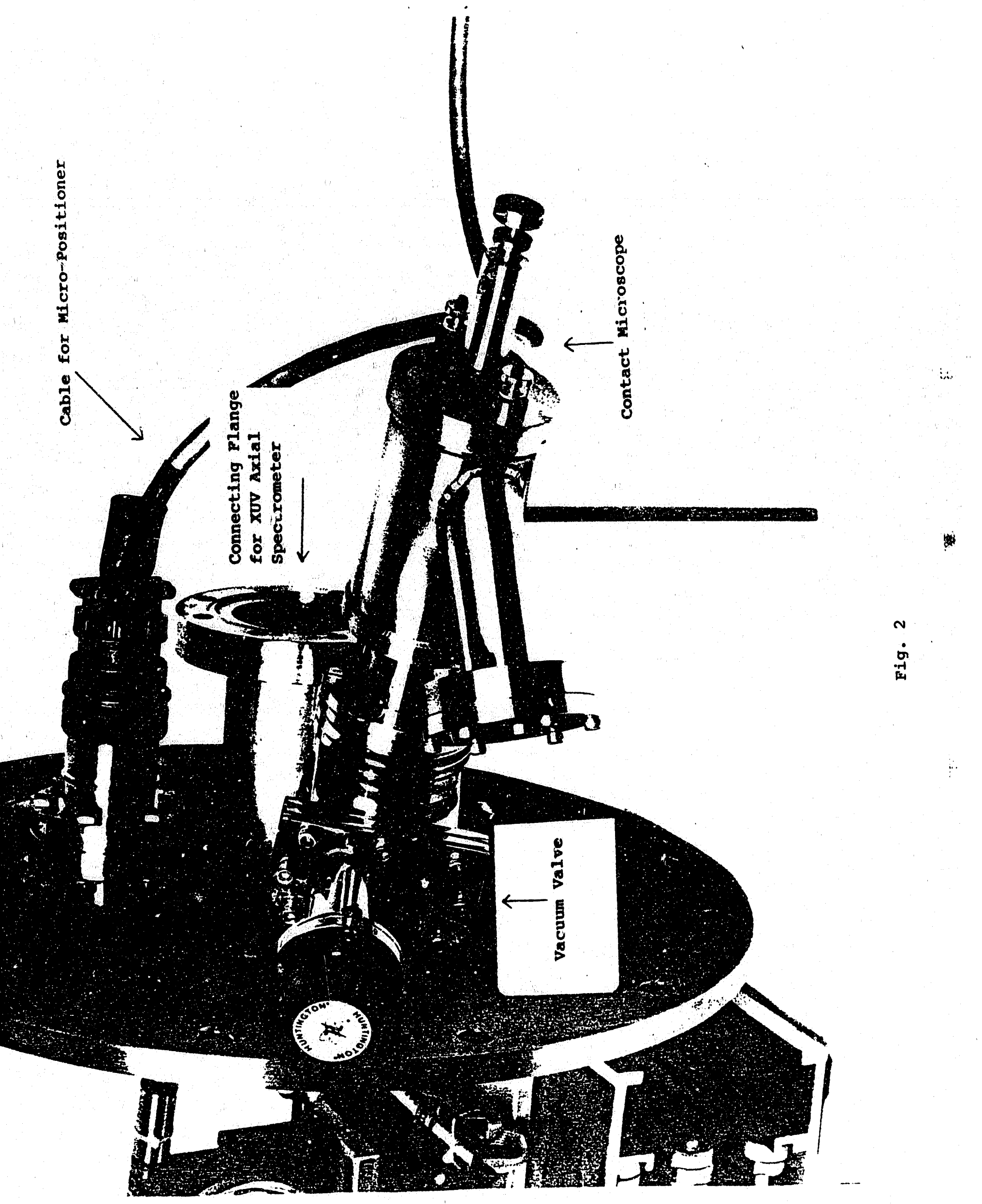




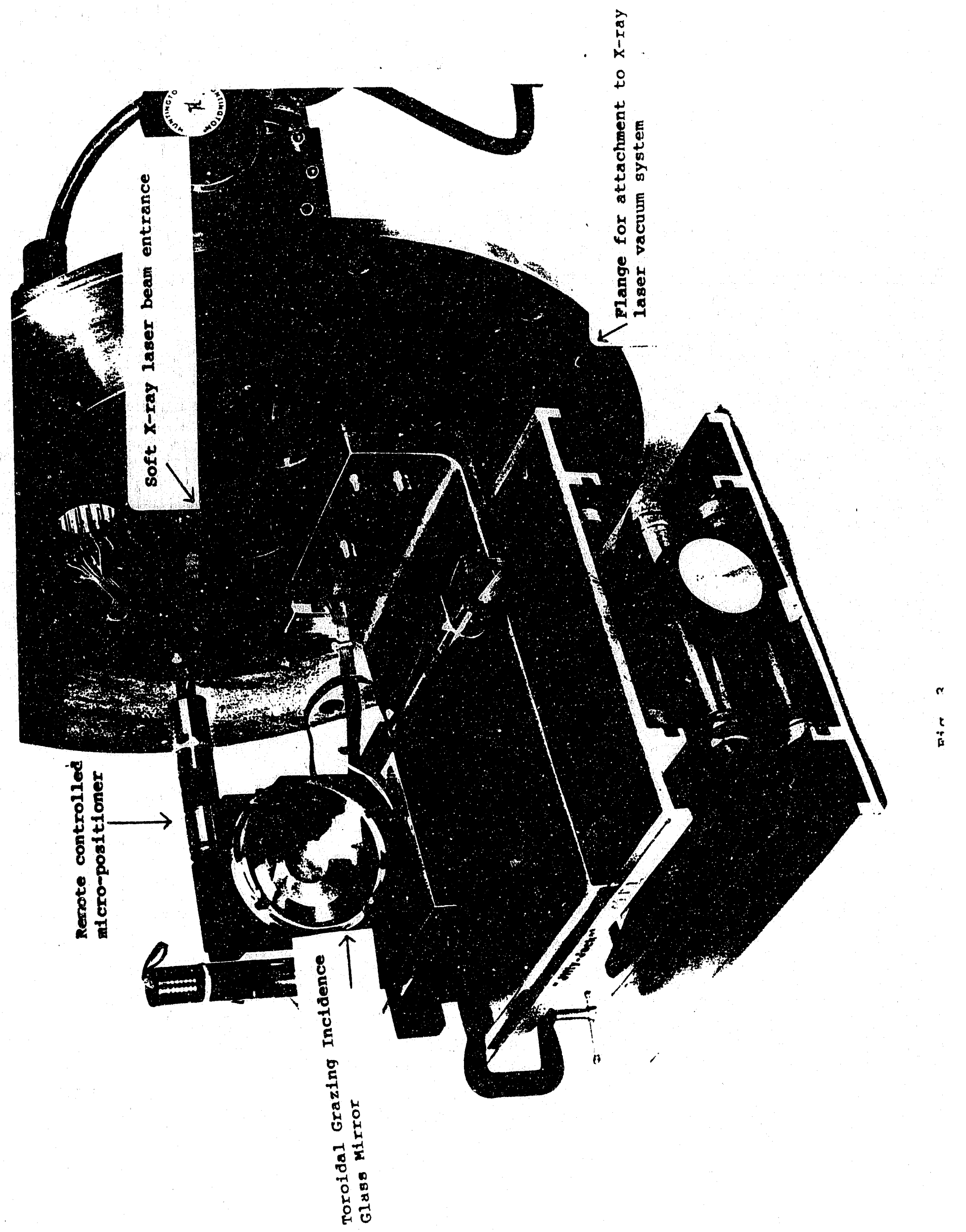




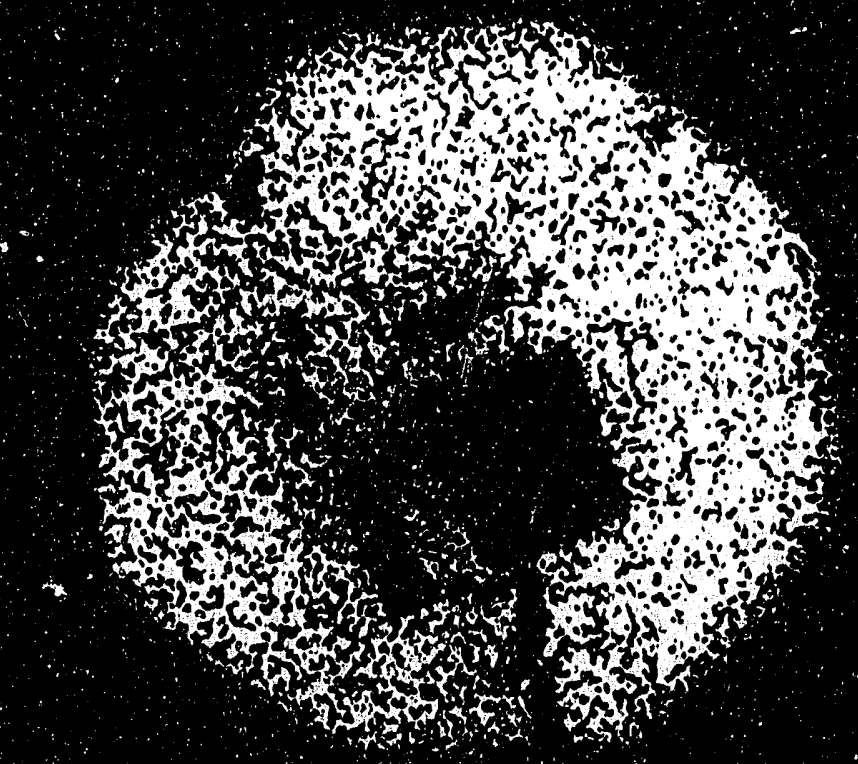
(a) filter (one laser shot)

Image of mesh on Kodak 101 with $\mathrm{Si}_{3} \mathrm{~N}_{4}$ window and $\mathrm{Al}$

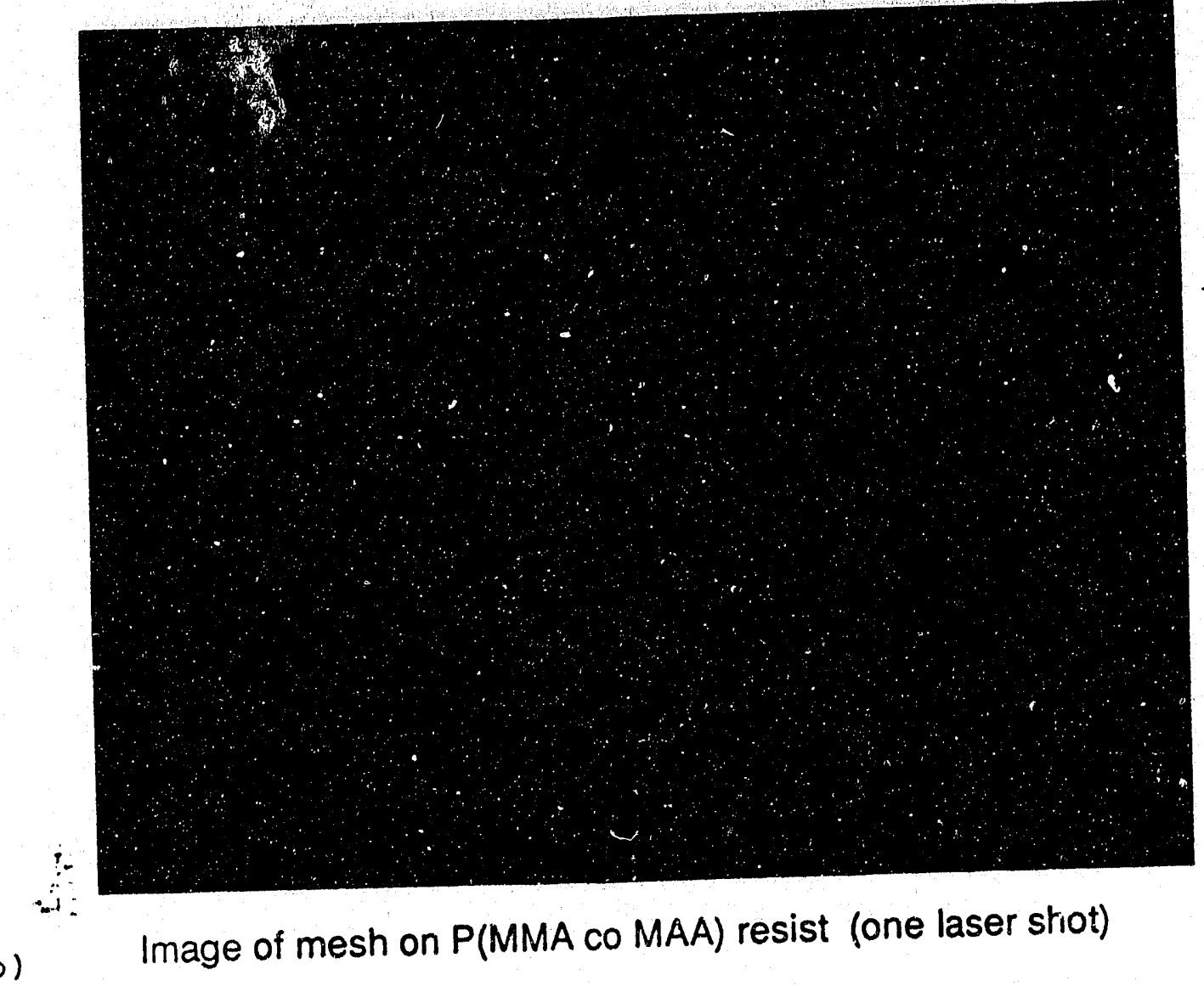




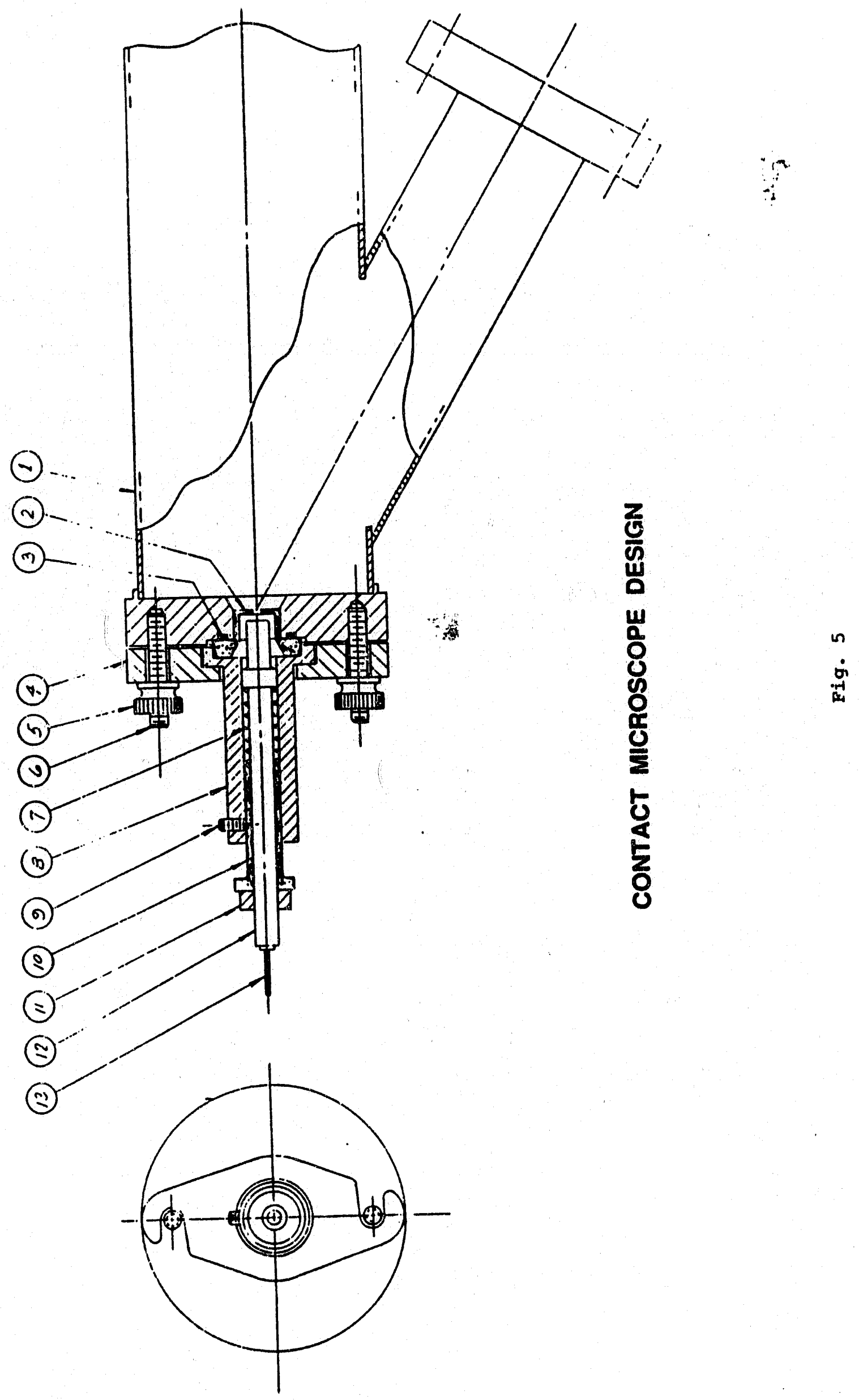


These may be seen in Fig. 4 where the advantage of the resist as a recording media is readily apparent. The resolution in the image recorded on film is limited to a few microns by grain structure.

The toroidal mirror was designed especially for our system to provide focussing of the $X$-ray laser beam on the microscope window. By moving the torodial mirror in or out of the laser beam, using an Oriel 3-axis remote controlled micro-positioner, the soft $X$ ray radiation is directed either to the microscope or to the axial soft $X$-ray spectrometer. (The spectrometer monitors the gain of the soft $X$-ray laser). A vacuum valve also has been added to the system between the microscope and the soft $X$-ray laser (not shown in Fig. 1) to allow the contact microscope to be accessed without venting the vacuum from the rest of the system.

In Fig. 5, is shown the PXL design of the contact microscope attached to the soft $X$-ray lase by a vacum tube 1 , and separated from the main vacuum chamber by the vacuum valve (not shown). The specimen is kept in place by holder 2 . The specimen chamber itself is shown schematically in Fig. 6 A window, made from SiliconNitride (Si3N4), $100 \mathrm{~nm}$ to $120 \mathrm{~nm}$ thick, isolated the biological specimen from the high vacuum region of the soft $X$-ray laser. The size of the window is $0.1 \mathrm{~mm}$ o. $0.1 \mathrm{~mm}$. A major consideration was the relationship between the window thickness, the size of the window, and the ability to withstand a one atmosphere pressure differential. It appears that the surface tension of the water associated with the biological specimen helps to support the $\mathrm{Si}_{3} \mathrm{~N}_{4}$ window. Initial studies based on the X-ray absorption curves of the silicon-nitride show that approximately $70 \%$ of the radiation intensity at $18.2 \mathrm{~nm}$ is absorbed by the window.

Let us go back to Fig. 5. The microscope had a number of specimen holders that can be prepared prior to the experimental run. The windows were glued into the holders using glyptol or another suitable adhesive. The specimen was placed behind the window and then the resist was placed behind the specimen. Microscope has the 
provision for the inclusion of a pin diode which can be mounted behind the window to aid in the alignment of the toroidal mirror with He-Ne laser. A viewing post had also been added to allow for a direct visible view during setup and alignment.

The specimen were recorded on resist by placing it directly onto the resist and then making a transmission exposure. To have the specimen as close as possible to the resist, live cells were growing directly on the resist.

PMMA was used for work reported by Rosser, Sayre, and Kirz ${ }^{2}$, and a theoretical resolution in the range of $10 \mathrm{~nm}$ was predicted for this material. The material is basically a very pure plastic similar to lucite. The $\mathrm{X}$-ray radiation weakens the bonds proportionally to the intensity passing through the biological specimen. When the resist is processed in a solvent, a relief of the biological specimen is formed with the depth proportional to the density of the biological material of the specimen. The resist can then be read using an electron microscope either in the transverse (through the resist) mode or with a scanning electron microscope (SEM) after the resist is coated with gold. One consideration was that the resist has to be tramsparent to visible light and of good optical quality when it is used in the Composite Optical/X-ray Laser Microscope because the specimen had to be viewed through the resist.

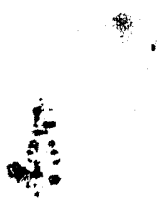


III. Composite Optical/X-Ray Laser Microscope (COXRALM) and Images of Biological Cells

COXRALM was conceived in Phase $I$ of the project in collaboration with Profs. J. Hirschberg and E. Kohen at the University of Miami. The conceptual design of COXRALM was described in Ref. 3 and is iliustrated in Figs.7 and 8. COXRALM was designed to greatly enhance the usefulness at a simple conact microscope and be a convenient, powerful and practical tool for biologists. It is a highly desirable for biologists to be able to select and manipulate a particular cell and view it in a conventional light microscope before exposure in a contact Soft X-ray Mic cope. That way, features aprarent in both the conventional and Sort $X$-ray image form a bridge to relate the two technologies and provide a basis against which new features, observable only in the $X$-ray image, can be interpreted. COXRALM is a combination of an inverted Leitz light microscope and a Soft $X$-ray Laser contact micros/ope. The light microscope is to be used to select and manipulate cells before exposure by the soft $x$-ray Laser. The construction of COXRALM was essentially complete in Phase II and the microscope was installed on the Soft X-ray Laser (see Fig. 9 and i0).

F.:

Our first studies of biological specimens were made with diatoms4. These are silicified skeletons of microscopic plankton algae and provide a high contrast object with submicon features. The diatoms were imaged on copolymer resist in vacuum and the resist developed for 1 minute in a mixture of equal parts methylisobutyl ketone and isopropanol. The image on the resist was viewed in on electron microscope and may be seen in Fig.11.

One feature that became apparent in this work is that the electron microscope could easily "write" a raster pattern in the resist if care was not taken to control the electron current and accelerating voltage. Electron beam damage to the resist could be avoided by limiting the time spent on the highest resolution scans. Future 
COMPOSITE X-RAY LASER MICROSCOPE (COXRALM)

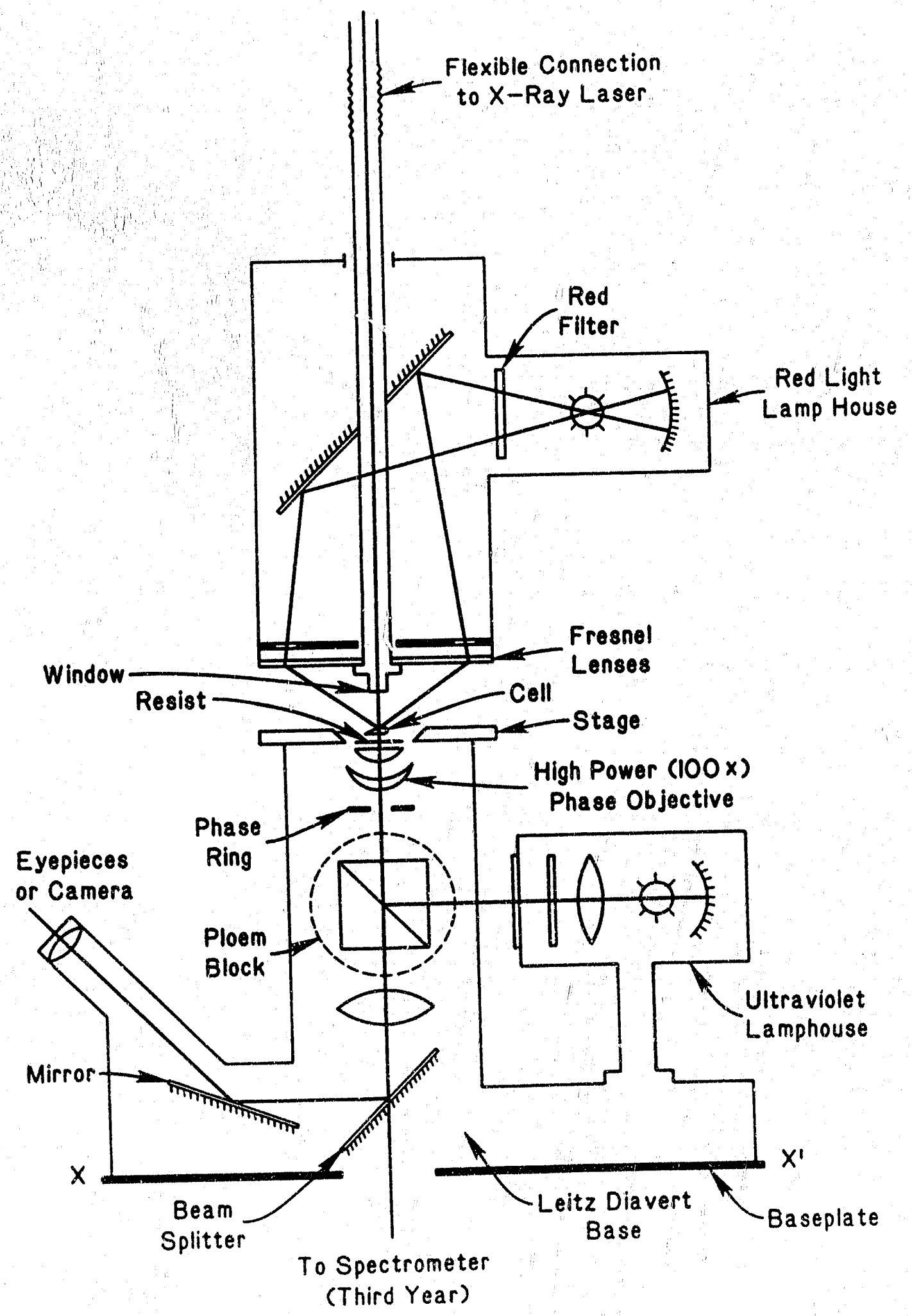


WUKALM IN VERTICAL POSITION

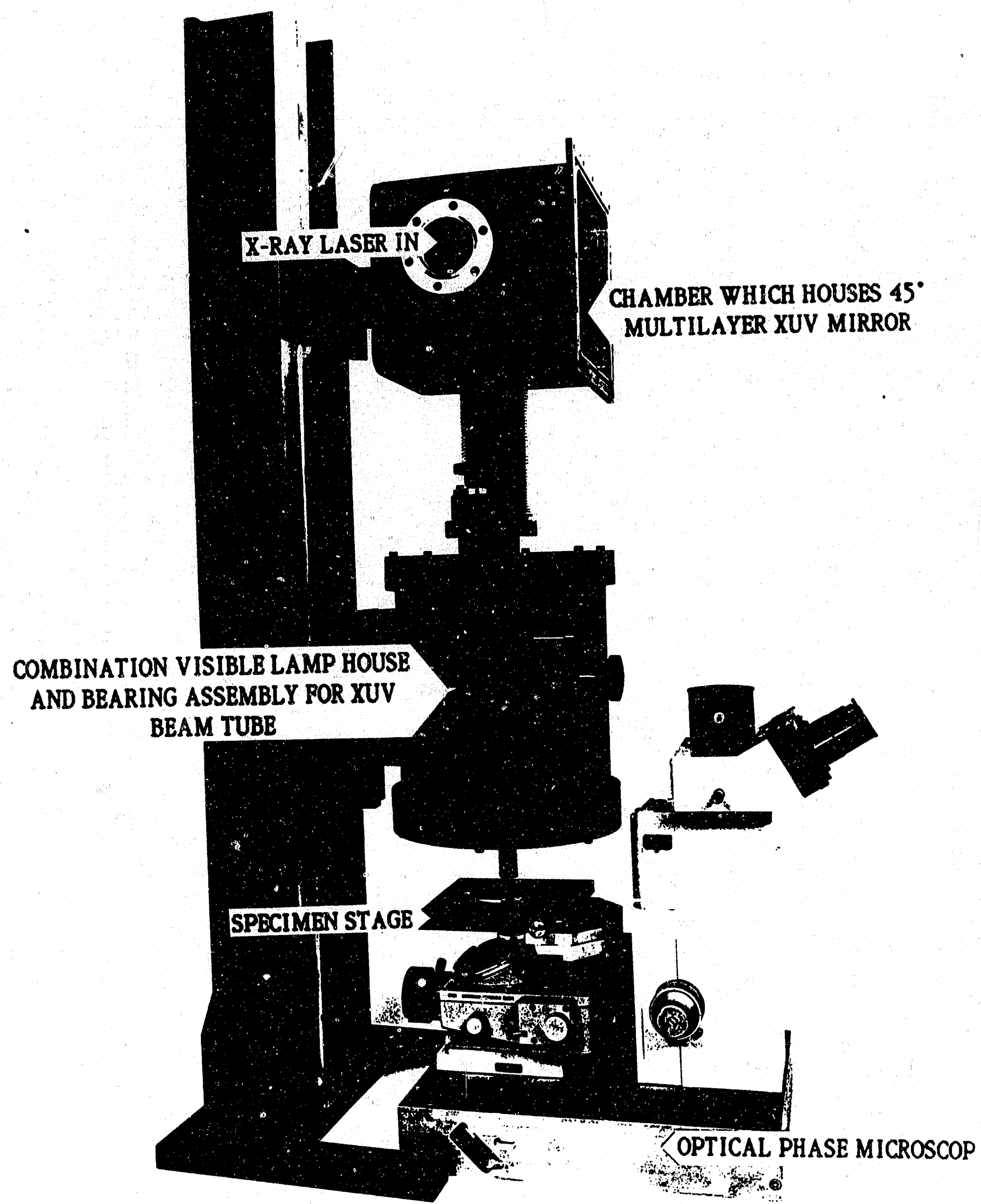

Fig. 8 


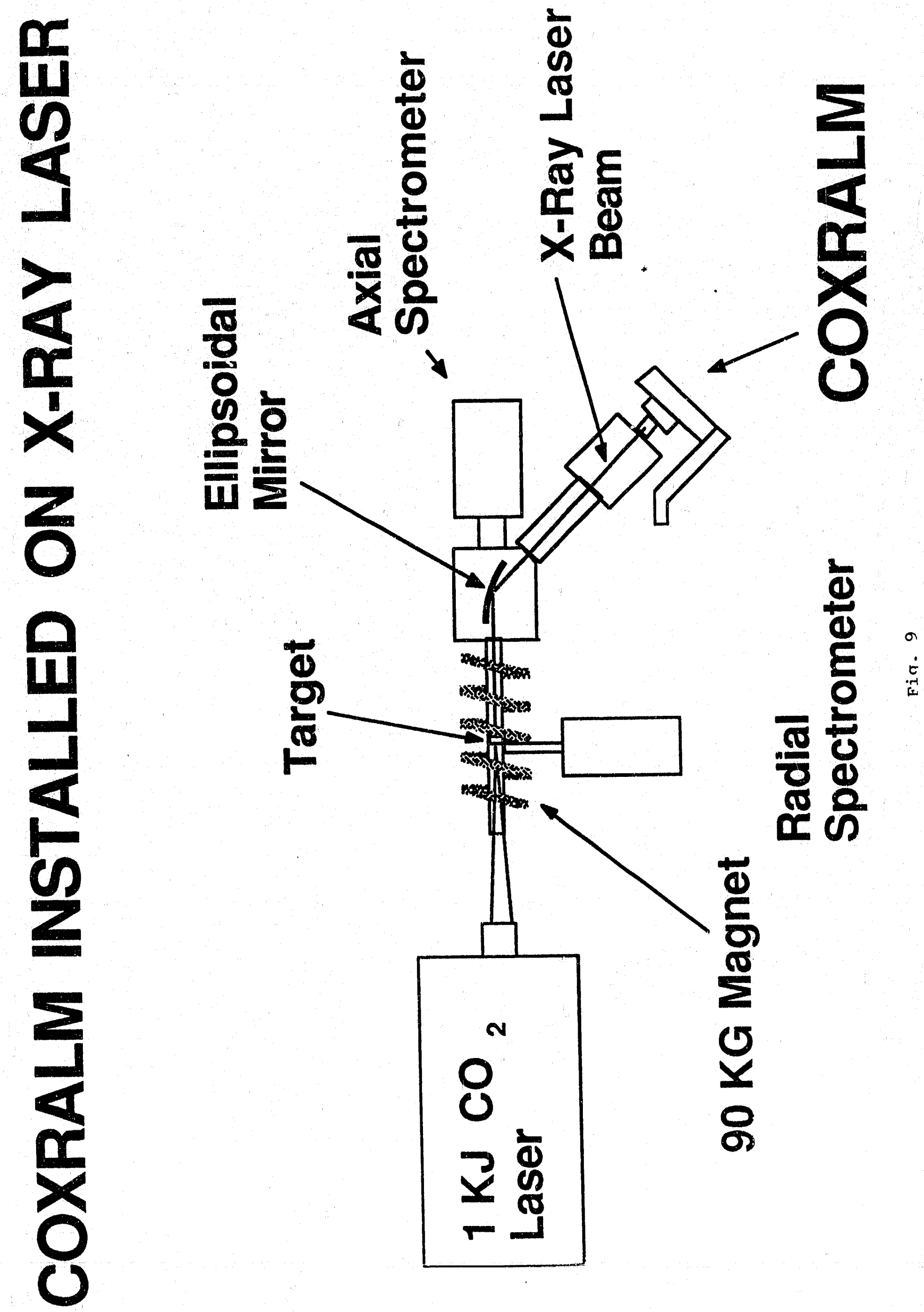




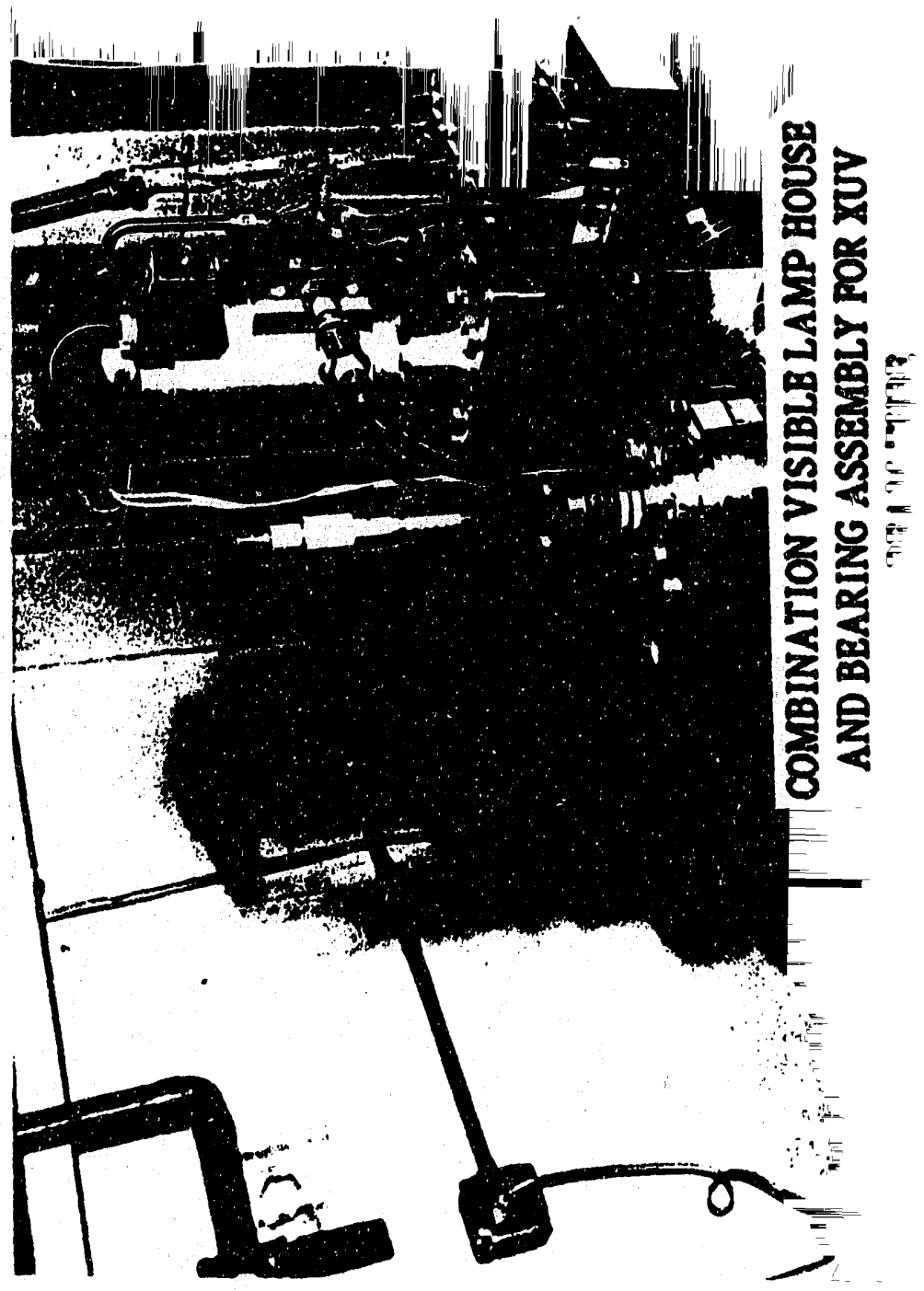


adoption of techniques of making damages resistant replicas of the resist alleviated this problem.

It was found that the photoresist PBS (polybutene-1-sulfone) was a factor of three more sensitive than the copolymer resist and was therefore used for subsequent exposures of biological specimens. The PBS resist was developed in methyl ethyl ketone ( 2 parts) and isopropanol (1 part) for 15 seconds. In initial experiments with biological specimens placed in contact with the resist, it proved difficult to remove the specimens from the resist after the exposure. This opened the possibility of confusion of remnants left on the resist with the relief image formed in the resist. In order to avoid this problem the biological specimens were mounted on a thin $(5 \mathrm{~nm})$ carbon film supported on a standard TEM grid. This was placed in contact with the resist for the exposure and could be easily removed and if necessary reused in later exposure.

We have been developing stronger links to the Biological Community. In addition to our association with Profs. J. Hirschberg and $\mathrm{E}$. Kohen at the University of Miami we have been actively collaborating with Prof. A. Gupta at Rutgers University, located nearby in New Brunswick. Prof. Gupta is an entomologist and is interested in the functional analogy of insect immune systems to the human immune system. This is the topic of considerable current interest in iology and since it involves the dynamic response of immune cells to foreign matter it is a natural subject for investigation by soft $X$-ray laser microscopy. One of the most primitive and hence simplest, immune system is found in the horseshoe crab (Limulus Polyphemus) where there is only one type of immune cell to fulfill several functions. A sample of blood from the horseshoe crab was dehydrated in an ethanol series and critical point dried using the standard procedure but was not fixed or stained and an exposure was taken with the soft X-ray laser contact microscope ${ }^{6}$. The resulting images viewed in an JEOL 840 scanning electron microscope are shown in Figure 12. The depressions apparent in the granulocyte are known to exhibit immune functions from work in 

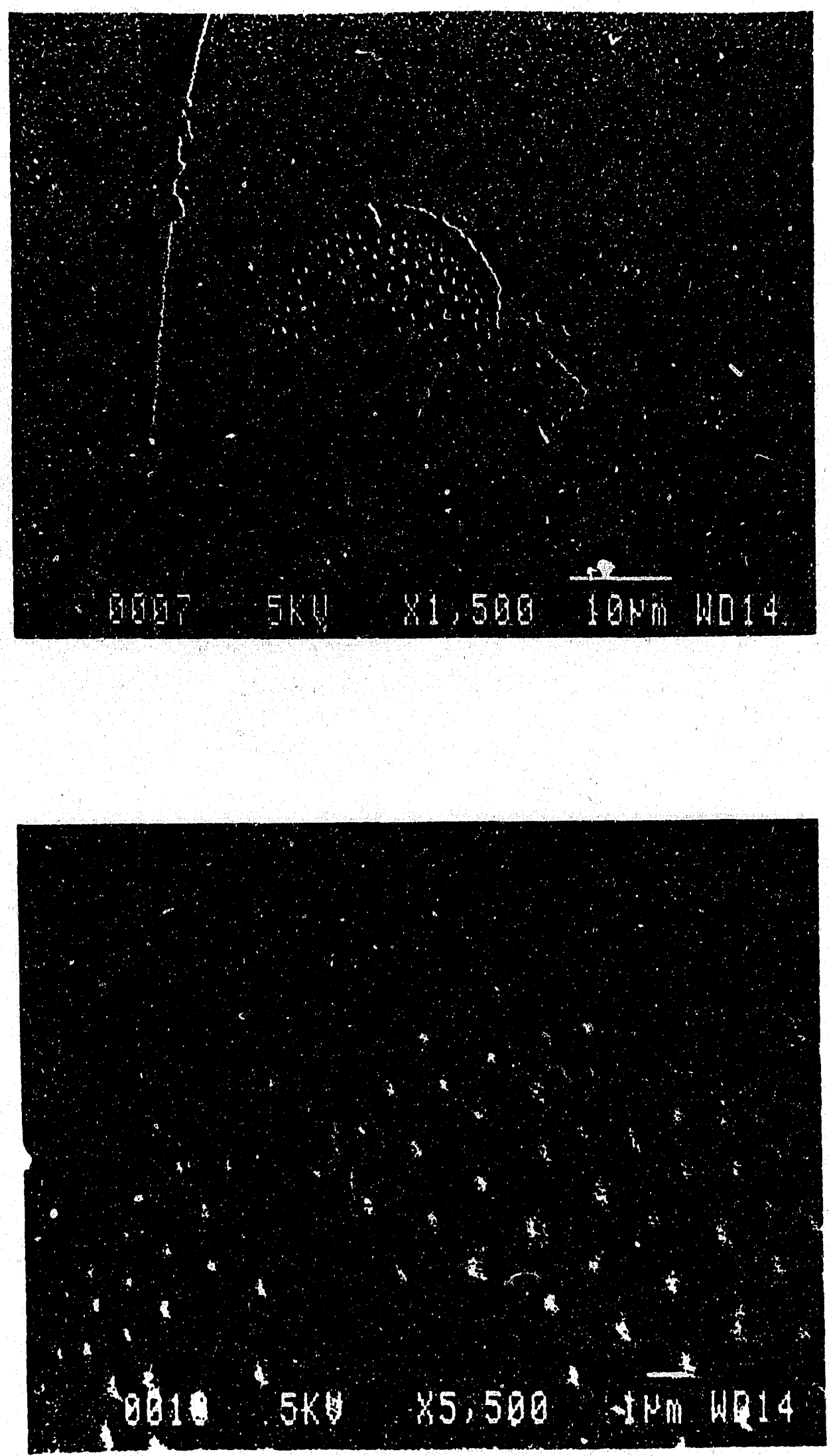

Fig. 11 

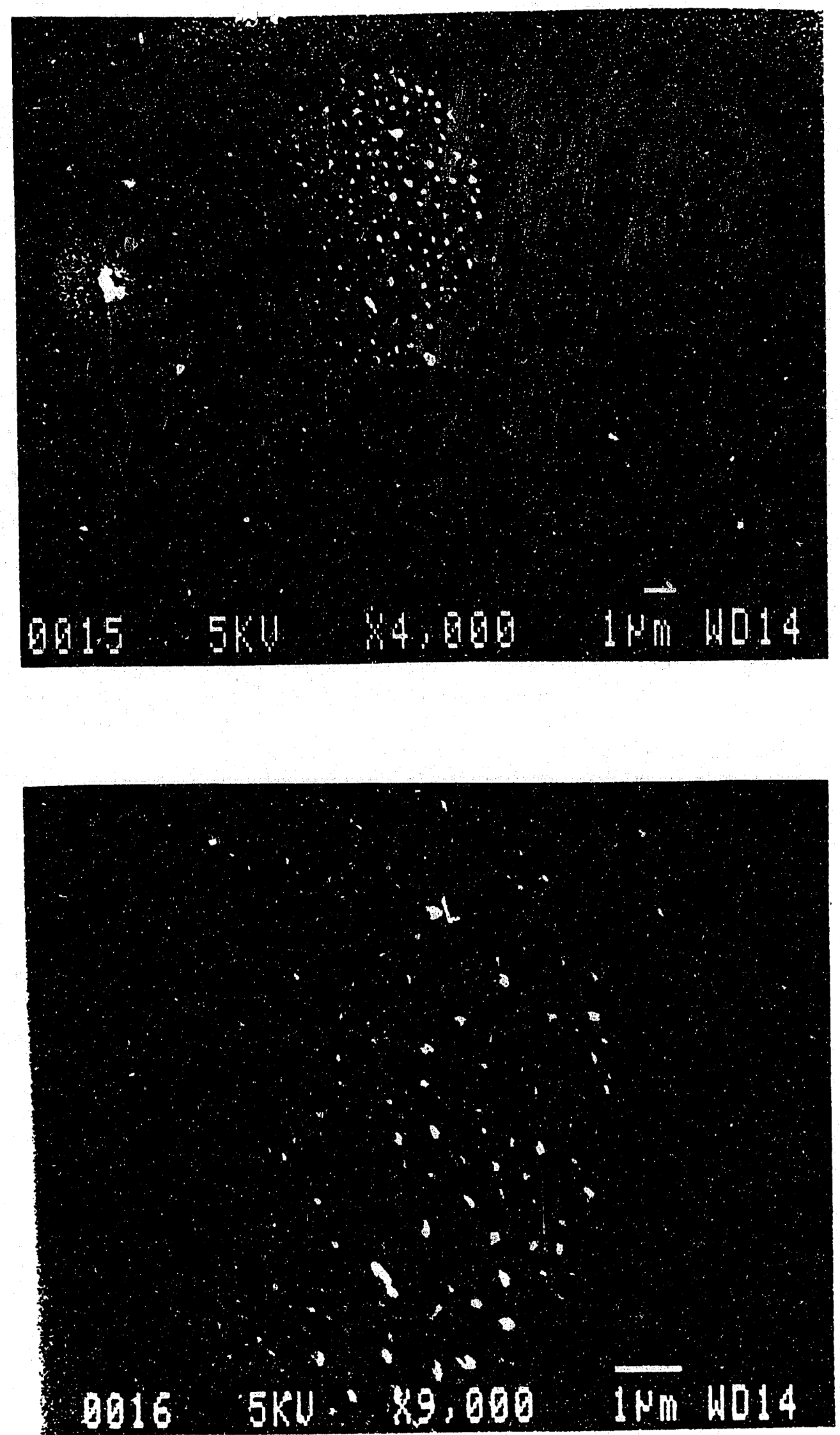

SEM image of an X-Ray Laser produced replica of a granulocyte from the animal Limulus Polyphemus (Horseshoe crab). This image was produced with a single shot of the X-Ray Laser. 
conventional electron microscopy. The smaller white feature covering the cell are new and were not apparent on images obtained in conventional electron microscopy. Their identity and function is presently unknowr..

One of the difficulties of examining photoresist relief patterns of biological images under a SEM is that replicas show little contrast, even with deep development. This is largely due to biological specimens typically having "soft edges" and slowly varying detail. The contrast of a SEM image formed by secondary electrons depends only on variations in surface slope; sharp edges show up clearly but not the gently undulating surfaces of biological specimens.

We have developed a technique that uses backscattered electrons from the SEM probe beam to produce a low magnification image with contrast proportional to the thickness of the resist. In this technique the resist has a gold underlay to increase the flux of backscattered electrons. The replica can be viewed clearly at low magnification with a very low beam current $\left(2 \times 10^{-12} \mathrm{~A}\right)$ and interesting features identified. The advantage here is that electron beam damage of the resist is negligible with such a low current, even over an lengthly viewing session. Good contrast was obtained in images formed with backscattered electrons of shallow relief patterns in a $150 \mathrm{~nm}$ resist backed with $100 \mathrm{~nm}$ of gold using a $5 \mathrm{keV}$, $10^{-12} \mathrm{~A}$ probe beam with a tilt of $25^{\circ}$ and $14 \mathrm{~mm}$ working distance. Features of interest can then be viewed at high magnification and high beam current $\left(2 \times 10^{-10} \mathrm{~A}\right)$ and fine details appears in the image formed by secondary electrons. The first high magnification images can be obtained before electron beam damage to the resist becomes evident.

This technique was used in the image of Hela cells shown in Fig. 13. This is an embedment-free section of a monolayer of extracted Hela cells (cultured human cervical cancer cells) on a carbon/formvar base6,7. The embedding material had been removed using the technique of Fey et. al (1986)8. 


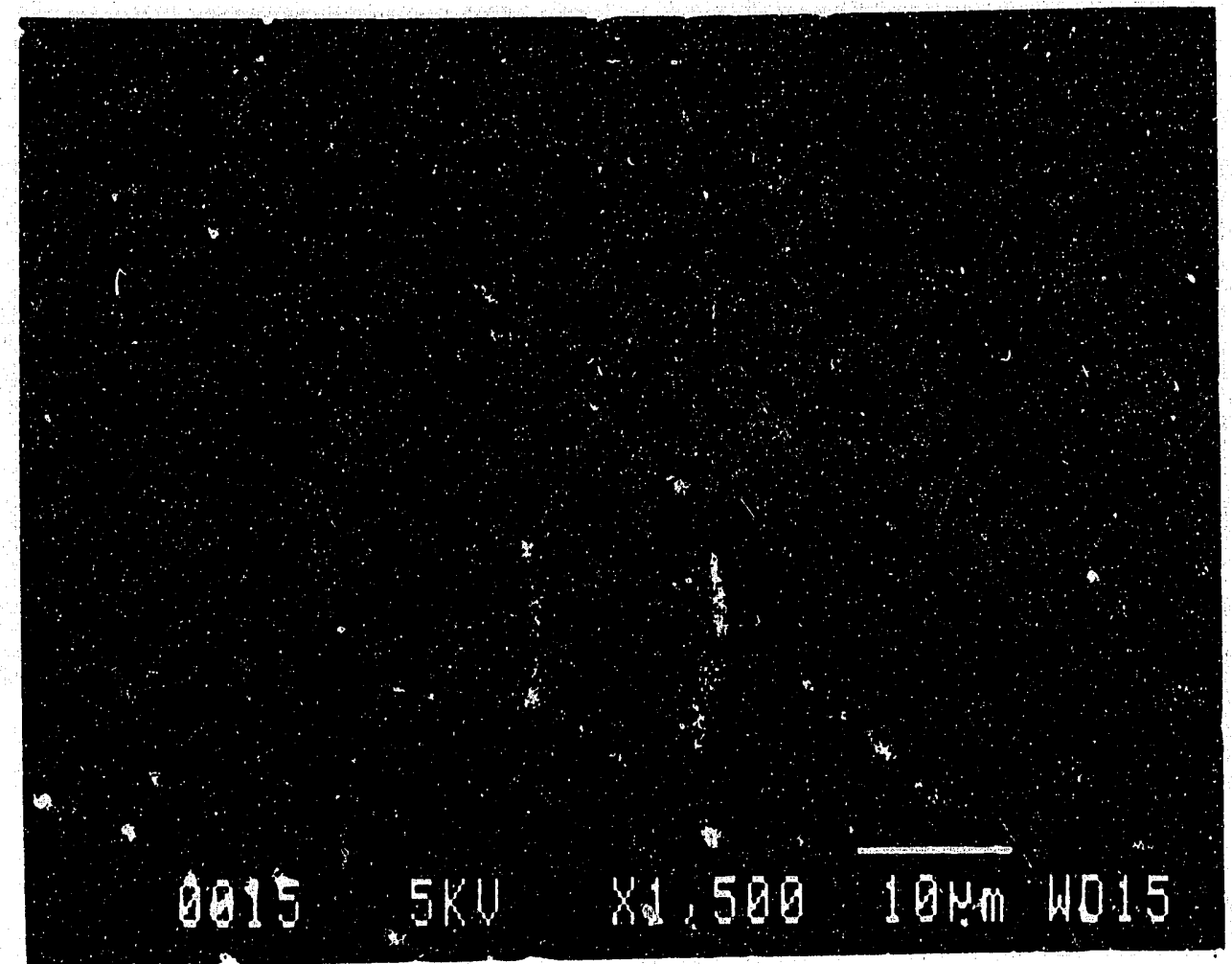

SEM IMAGE OF AN X-RAY LASER MICROSCOPE PRODUCED REPLICA OF AN EXTRACTED HELA-CALL MONOLAYER (HELEN LANE CERVICAL CANCER CELLS) 
IV. Imaging X-Ray Lasing Microscope (IXRALM)

The third year of the program (second year of Phase II) was devoted, as mentioned earlier, to the construction of Imaging X-Ray Microscope (IXRALM) and was conducted in parallel to the study of biological cells with COXRALM. The main advantage of IXRALM in comparison with contact microscopes is that the magnified image of the specimen may be recorded directly on a charge coupled device or photographic film of much higher sensitivity than photo-resist, thus reducing the $X$-ray exposure of the specimen. Possible artifacts introduced in the processing of photo-resist are also avoided.

The simplified scheme of IXRALM is presented in Fig. 14 and the picture of this microscope attached to X-ray laser is shown in Fig.15. In Fig. 14, one may see two principal imaying elements of the microscope: multilayer coated Schwarzschild condensor and zoneplate objective, providing magnification $\sim 100$. Because of sensitivity of the film or CCD array is $\sim 10^{6}$ time higher than photoresist, and although loosing about $10^{3}$ beam intensity on imaging optics, still overall gases in sensitivity of imaging microscope in comparison with contact microscope is $\sim 10^{3}$. This allows the "portable" $X$-ray laser with beam to use the intensity on the range $\sim 10 \mu \mathrm{J}$ instead of several $\mathrm{mJ}$ as in case of contact microscope.

IXRALM has build-in interferometric system based on matching Schwarzchild mirror, which allows to provide precise alignment of $X$-ray laser beam on Schwarzschild condensor. After IXRALM was build we find-out during testing experiments that alignment of condensor can be done with good precision. However we have forced major $c^{\prime}$ ifficulties with providing proper alignment of the zoneplate. Several months of trial-and-error experiments with zoneplate brings us to the conclusion that IXRALM was to be redesigned. In a new IXRALM version, the zoneplate is replaced by very high quality multilayer Schwarzschild objective. The microscope is constructed and ready for alignment and to begin 
testing. This work (Phase III) will be conducted by PXL in cooperation with Princeton University PPL during the next 3-4. months. 
Imaging X-ray Microscopo

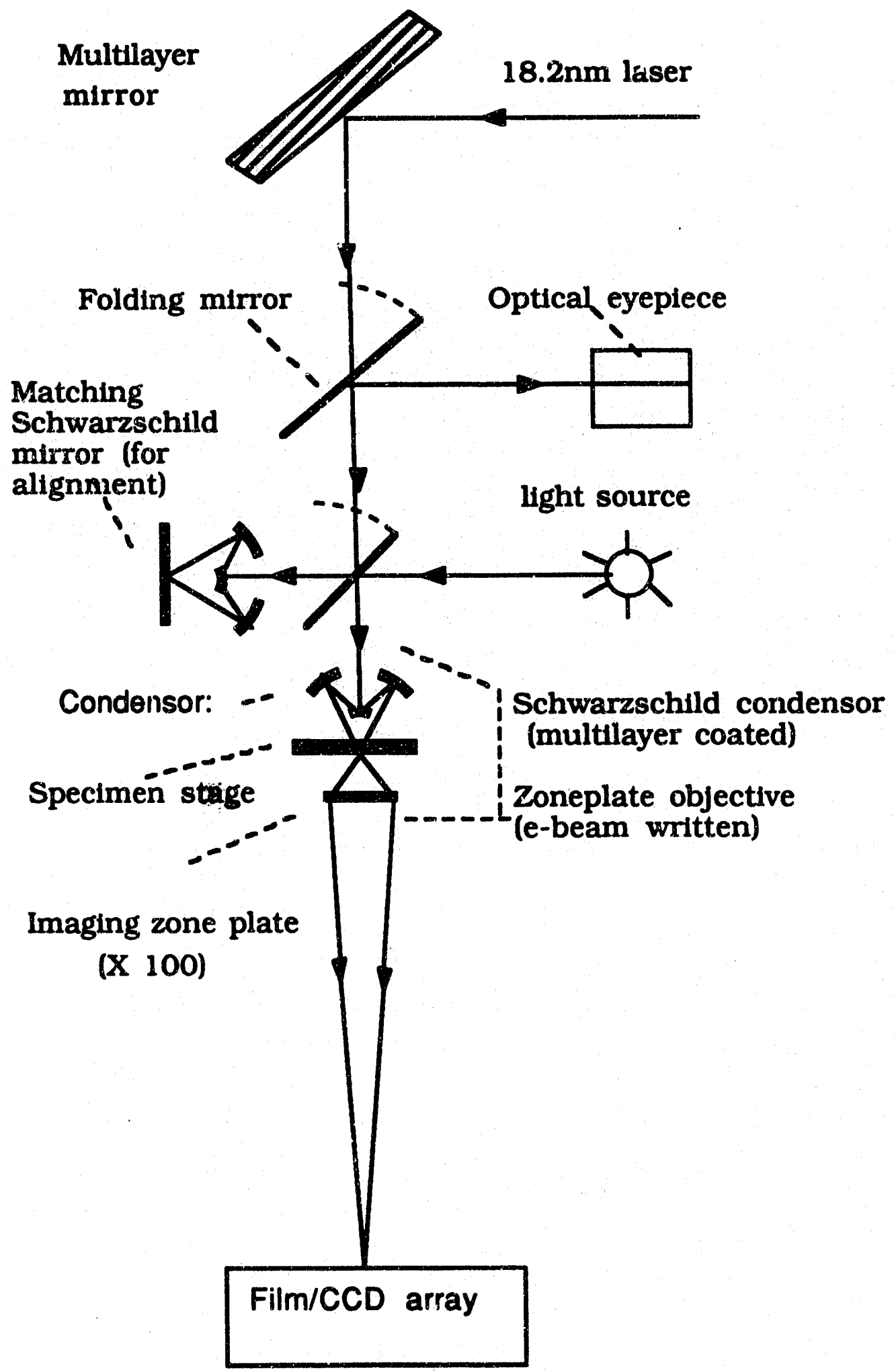




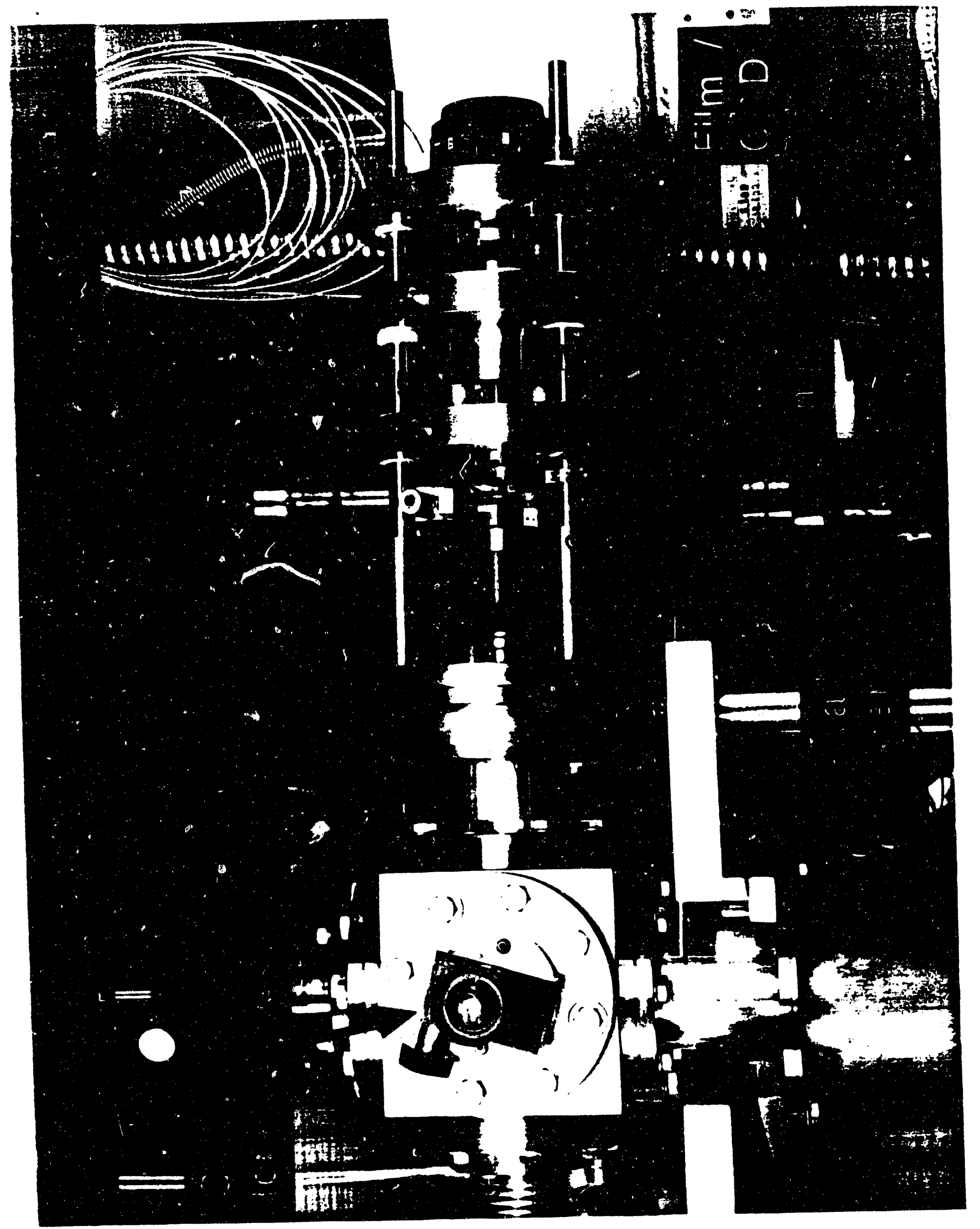


References

1PXL Progress Report on Soft X-Ray Laser Microscope (Phase I), June 1987.

2R. Rosser, J. Kirz, D. Sayre, et al., J. Microsc. 138, 311 (1985).

3PXL Progress Report on Soft X-Ray Laser Microscope (Phase IIFirst Year), August 1988.

4D S. DiCicco, et al., "Contact Microscopy with Soft X-Ray Laser", in Proc. of Conf. on Short Wavelength Coherent Radiation, Cape Cod, Mass., Sept. 1988.

5P. C. Cheng, G. J. Jan (Eds.), X-Ray Microscope, Springer-Verlag, Berlin, (1987).

6C. H. Skinner, et al., J. Microsc. 159,51 (1990).

7S. Suckewer and C. H. Skinner, Science 247, 1553 (1990).

8E. G. Fey, et al., J. Cell. Biol. 102, 1654 (1986). 
13

APPENDIX

Attached publications on X-Ray Laser microscopy conducted by PXL in cooperation with PPL.

Reprint + Prepsint removed 

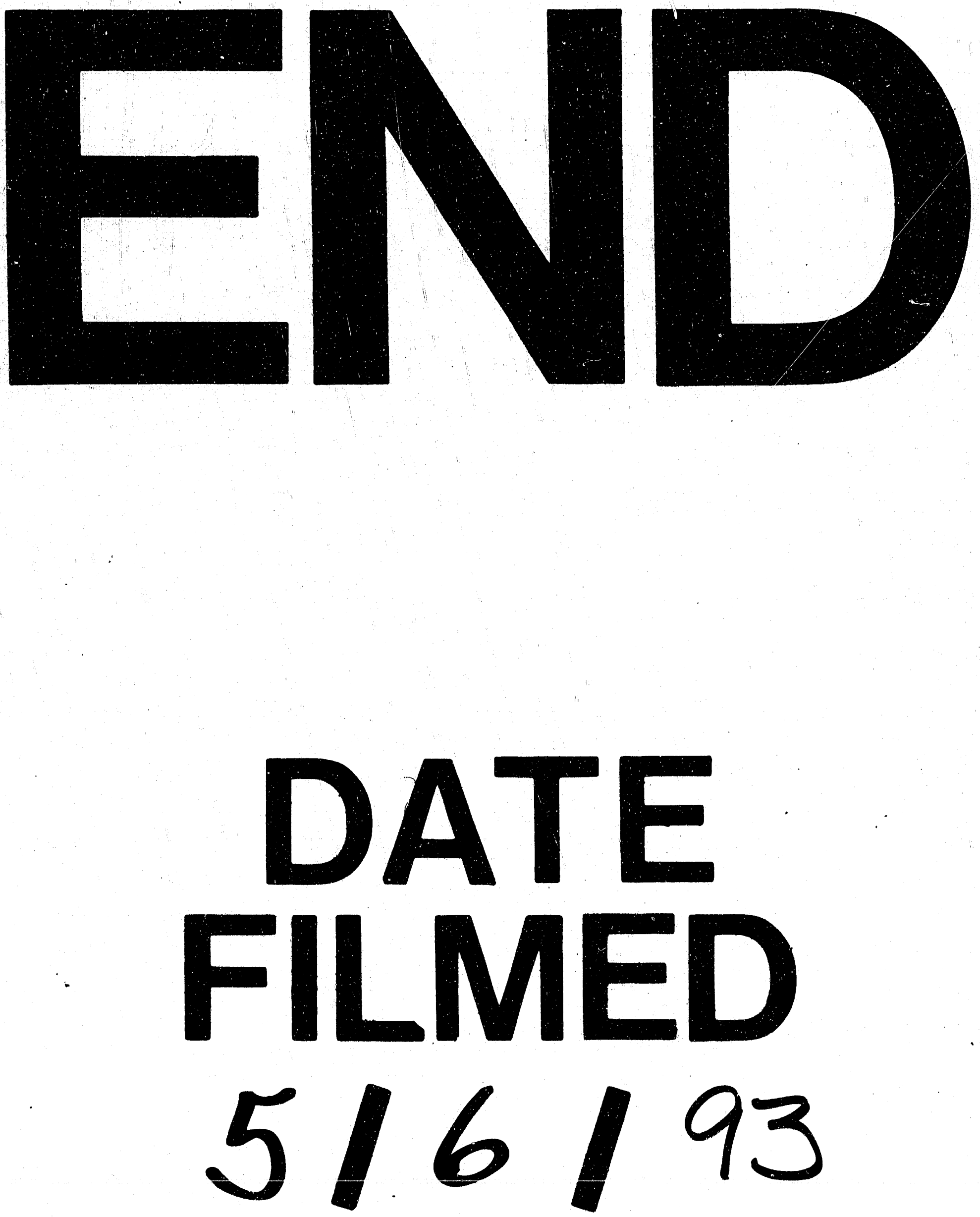
